\title{
Ablation of the MiR-17-92 MicroRNA Cluster in Germ Cells Causes Subfertility in Female Mice
}

\author{
Jian Wang ${ }^{\mathrm{a}}$ Bo Xu $\mathrm{u}^{\mathrm{a}}$ Geng G. Tian ${ }^{\mathrm{a}}$ Tao Sun ${ }^{\mathrm{b}}$ Ji Wu $\mathrm{U}^{\mathrm{a}, \mathrm{c}, \mathrm{d}}$ \\ aKey Laboratory for the Genetics of Developmental \& Neuropsychiatric Disorders (Ministry of \\ Education), Bio-X Institutes, Shanghai Jiao Tong University, Shanghai, 'bSchool of Life Sciences and \\ Biotechnology, Shanghai Jiao Tong University, Shanghai, 'Key Laboratory of Fertility Preservation and \\ Maintenance of Ministry of Education, Ningxia Medical University, Yinchuan, dShanghai Key Laboratory \\ of Reproductive Medicine, Shanghai, China
}

\section{Key Words}

MicroRNA • MiR-17-92 cluster • Subfertility • Apoptosis

\begin{abstract}
Background/Aims: Oogenesis is a highly complex process that is intricately regulated by interactions of multiple genes and signaling molecules. However, the underlying molecular mechanisms are poorly understood. There is emerging evidence that microRNAs contribute to oogenesis. Here, we aimed to investigate the role of miR-17-92 cluster in regulating oogenesis. Methods: The miR-17-92 cluster was genetically ablated in germ cells of female mice by applying the Cre-loxp system for conditional gene knockout. Mating experiment, superovulation and histological analysis were used to assess the fertility of the model female mice. TUNEL assay was used to identify apoptotic cells in ovaries. The expression level of apoptosis- and follicular atresia- related genes was evaluated by qRT-PCR. Western blotting was performed to detect protein expression. Bioinformatics software and dual luciferase reporter assay were applied to predict and verify the target of miR-17-92 cluster. Results: Deletion of miR-17-92 cluster in germ cells of female mice caused increased oocyte degradation and follicular atresia, perturbed oogenesis, and ultimately led to subfertility. Genes involved in follicular atresia and the mitochondrial apoptotic pathway were obviously up-regulated. Furthermore, we verified that miR-19a regulated oogenesis at the post-transcriptional level by targeting Bmf in the ovaries of miR-17-92 cluster conditional knockout female mice. Conclusion: The miR-17-92 cluster is an important regulator of oogenesis. These findings will assist in better understanding the etiology of disorders in oogenesis and in developing new therapeutic targets for female infertility.
\end{abstract}

Ji Wu

and Tao Sun

\section{KARGER}

Key Laboratory for the Genetics of Developmental \& Neuropsychiatric Disorders, Bio-X Institutes, Shanghai Jiao Tong University, No. 800 Dongchuan Road, Minhang District, Shanghai (China); Tel. +86-21-34207263, E-Mail jiwu@sjtu.edu.cn, taosun11@sjtu.edu.cn 


\section{Cellular Physiology Cell Physiol Biochem 2018;45:491-504 \begin{tabular}{ll|l} 
DOI: 10.1159/000487028 & O 2018 The Author(s). Published by S. Karger AG, Basel \\
www.karger.com/cpb
\end{tabular} \\ Wang et al.: Ablation of the MiR-17-92 in Germ Cells Causes Subfertility}

\section{Introduction}

As a public health problem, infertility affects as many as 48.5 million couples worldwide [1]. Disorders in oogenesis are the main cause of female infertility. Such disorders are caused by many risk factors, including genetic, epigenetic and environmental ones, making investigation of the pathophysiology of these disorders extremely challenging. Oogenesis is a highly complex, yet robust process that is intricately regulated by interactions of multiple genes and various signaling molecules. However, the underlying molecular mechanisms remain largely obscure.

MicroRNAs (miRNAs)—small, endogenous, noncoding RNA molecules-are known to negatively regulate gene expression either by translational suppression or by destabilizing messenger RNA (mRNA) molecules [2-4]. Currently, many miRNAs have been identified and characterized in the ovaries of various mammalian species, including mice [5, 6], humans [7], cows [8], goats [9], sheep [10] and pigs [11]. Dicer is the ribonuclease III used in the biogenesis of mature functional miRNAs [12]. Global depletion of miRNA functions using Dicer ablation results in increased follicular atresia [13] and lower ovulation rate [12] in Dicer conditional knockout (cKO) mice. miRNAs are suggested to play fundamental roles in the formation of primordial follicles, follicular recruitment and selection, follicular atresia, oocyte-cumulus cell interaction, granulosa cell function, and luteinization [14].

A typical multifunctional gene cluster, miR-17-92, encodes six miRNAs (miR-17, 18a, 19a, 20a, 19b, and 92a). Emerging evidence has shown that this cluster is essential for many developmental and pathogenic processes [15], such as type B lymphopoiesis [16], lung development [16], heart development [17], neurogenesis [18, 19] and tumorigenesis [20]. It is involved in the processes of cell proliferation, apoptosis, differentiation and metastasis. Several research groups have reported that the miR-17-92 cluster contributes to spermatogenesis [21-27]. He et al. found that miRNA-20 and miRNA-106a are preferentially expressed in mouse spermatogonial stem cells (SSCs). miRNA-20 and miRNA-106a regulate SSCs renewal at the post-transcriptional level by targeting STAT3 and Ccnd1 [26]. In 2010, Björk et al. verified that miR-18 targets heat shock transcription factor 2 in spermatogenesis [24]. In 2011, Tong et al. reported that, compared with wild-type (WT) controls, male germ cell-specific miR-17-92 knockout (KO) mice exhibited small testes, lower numbers of epididymal sperm, and mild defects in spermatogenesis [25]. Similarly, in 2016, Xie et al. showed that targeted disruption of miR-17-92 in the testes of adult mice resulted in severe testicular atrophy, many empty seminiferous tubules, and depressed sperm production [27]. They further demonstrated that dysregulated mTOR signaling contributed to the pathogenesis of testicular anomalies [27]. However, little is known about the functions of miR-17-92 in the regulation of oogenesis.

Here, we genetically ablated the miR-17-92 cluster in the germ cells of female mice by applying the Cre-loxp system for conditional gene KO (cKO). These cKO mice displayed much reduced fecundity with increased follicular atresia and lower ovulation numbers compared with controls. Moreover, genes involved in follicular atresia and the mitochondrial apoptotic pathway were obviously upregulated. We further verified that miR-19a regulated oogenesis at the post-transcriptional level via targeting Bmf (BCL2-modifying factor) in the ovaries of miR-17-92 cKO female mice. Our results demonstrate that the miR-17-92 cluster is an important regulator controlling oogenesis.

\section{Materials and Methods}

\section{Mice}

Floxed miR-17-92 transgenic mice (referred to as miR-17-92 floxfflox mice) [16] were supplied by the laboratory of Dr. Tyler Jacks, MIT, USA. The Ddx4-Cre mice carrying the Asp-Glu-Ala-Asp (DEAD) box polypeptide $4(D d x 4)$ promoter-mediated Cre recombinase, which is specifically expressed in germ cells [28], were purchased from the Model Animal Research Center of Nanjing University, P. R. China. To delete

\section{KARGER}




\section{Cellular Physiology Cell Physiol Biochem 2018;45:491-504

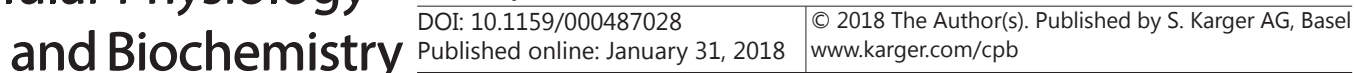 \\ Wang et al.: Ablation of the MiR-17-92 in Germ Cells Causes Subfertility}

the miR-17-92 cluster in mouse germ cells, first, male Ddx4-Cre mice were bred with female miR17-92 flox/flox mice. Then, male offspring with the genotype miR-17-92 flox/+; Ddx4-Cre were bred with female miR-17-92 flox/flox mice to generate miR-17-92 flox/s; Ddx4-Cre mice, called here miR17-92 cKO mice. C57BL/6 mice were purchased from SLAC Laboratory Animal Co., Shanghai, P. R. China. All procedures involving animals were approved by the Institutional Animal Care and Use Committee of Shanghai Jiao Tong University, and were conducted in accordance with the National Research Council Guide for the Care and Use of Laboratory Animals.

\section{Mouse Genotyping}

For mouse genotyping, tail-tip biopsies were subjected to polymerase chain reaction (PCR) amplification using a primer pair specific for Cre to amplify a 240-bp Cre fragment, primer pair F4/R5 to amplify 255-bp (miR-17-92 wide type allele, miR-17-92 $)$ and/or the 289-bp (miR-17-92 floxed allele, miR17-92 flox fragment, and primer pair F4/AV145 to amplify the 441-bp miR-17-92 deleted allele fragment (miR-17-92 ${ }^{\Delta}$ ) (Table 1).

\section{In Situ Hybridization}

miRNA expression patterns were detected by in situ hybridization (ISH) on paraffin wax sections according to previously published methods with modifications [29], using digoxigenin (DIG)-labeled locked nucleic acid (LNA) probes. Briefly, after being treated with proteinase $\mathrm{K}$, sections were hybridized with LNA probes diluted with Enzo ISH buffer (Enzo Life Sciences, USA) at $37{ }^{\circ} \mathrm{C}$ overnight. Then, the slides were washed in 0.2 x saline-sodium citrate with $2 \%$ bovine serum albumin at $4{ }^{\circ} \mathrm{C}$ for $5 \mathrm{~min}$. Subsequently, a horseradish peroxidase (HRP)-based signal amplification system was hybridized to the probes before color development with 3, 3'-diaminobenzidine tetrahydrochloride. Sections were then counterstained with hematoxylin. Images were obtained with a Leica DM2500 microscope (Leica, Germany). Sections hybridized without probes were used as negative control.

\section{Superovulation}

For superovulation, adult miR-17-92 cKO female mice were injected intraperitoneally with pregnant mare serum gonadotropin (Sansheng, China; $10 \mathrm{IU}$ ) and human chorionic gonadotropin (Sansheng, China; $10 \mathrm{IU}) 48 \mathrm{~h}$ apart. Mice were euthanized by cervical dislocation and metaphase II oocytes were collected from oviducts $14 \mathrm{~h}$ post human chorionic gonadotropin injection. miR-17-92 flox/flox females at the same age as the miR-17-92 cKO females were used as controls.

\section{Fertility Assessment}

Eight- to 12-week-old miR-17-92 cKO female mice were subjected to a continuous mating study. Two female mice were housed with one 8- to 10-week-old known fertile WT male mouse. The numbers of offspring per litter were recorded. Female miR-17-92 flox/flox mice were used as controls.

\section{Histology}

Mouse ovaries were fixed with 4\% paraformaldehyde (PFA) in phosphate-buffered saline (PBS) at 4 ${ }^{\circ} \mathrm{C}$ overnight, embedded in paraffin wax, and sectioned at $6 \mu \mathrm{m}$. Slides used for histological analysis were stained with hematoxylin. Images were obtained with a Leica DM2500 microscope and a Leica DFC 550 digital camera. Follicle numbers (including atretic follicles) were quantified as described [30, 31].

Terminal Deoxynucleotidyl Transferase dUTP Nick End Labeling (TUNEL) Assay

To identify apoptotic cells in ovaries, assays were performed using the TUNEL BrightRed Apoptosis Detection Kit (Vazyme, China) on $6 \mu \mathrm{m}$ paraffin wax sections, according to the manufacturer's instructions. Slides incubated in the absence of terminal deoxynucleotidyl transferase were used as negative controls. 


\section{Cellular Physiology Cell Physiol Biochem 2018;45:491-504 and Biochemistry Published online; January 31, 2018 \begin{tabular}{l|l} 
DOI: 10.1159/000487028 2018 The Author(s). Published by S. Karger AG, Basel \\
www.karger.com/cpb
\end{tabular} \\ Wang et al.: Ablation of the MiR-17-92 in Germ Cells Causes Subfertility}

Quantitative Real-Time Reverse Transcription PCR (qRT-PCR)

Total RNA was isolated from the ovaries of WT, controls and miR-17-92 cKO mice using Trizol reagent (Invitrogen, USA) according to manufacturer's instructions. Reverse transcription was performed using HiScript II Q RT SuperMix for qPCR (+gDNA wiper) kit (Vazyme, China). qRT-PCR was performed using FastStart Universal SYBR Green Master Mix kits (Roche, Germany) on an ABI PRISM 7500 system (Applied Biosystems, USA) according to the procedure used by Sun et al. [32]. The $2^{-\Delta \Delta C t}$ method was used to calculate the relative expression of genes within the ABI 7500 System Software (V2.0.4) and the gene expression levels were normalized to GAPDH or U6. The primers used for qRT-PCR are shown in Table 2.

\section{Luciferase Assay}

The 3'-untranslated region (UTR) of Bmf containing the binding sites for miR-19a was amplified by PCR using the following primers: forward (F), 5'-ACG TCT CGA GCT CCC TTT AGC TTT CAG CTA GG- 3'; and reverse (R), 5'-ATT TGC GGC CGC CAC CTA GCA AGG TTG CTG AAG3 '. The PCR fragment was inserted into the psiCHECK $^{\mathrm{TM}}$-2 vector (Promega, USA) by digestion with Xhol and NotI. For mutagenesis of miRNA, three nucleic acids from the seed sequence of the miR-19a mature sequence were mutated using QuikChange II Site-Directed Mutagenesis kit (Agilent Technologies, USA). The primer used for mutagenesis was: miR-19a-mut, 5'-GCA GCC CTC TGT TAG TTT TGC ATA GTA GAG TAC AAG AAG AAT GTA GTT CTA CTA ATC TAT CAA AAC TGA TGG TGG CCT GC-3'. To generate miRNA expression constructs, miR-19a fragment was inserted into the pcDNA3.1 as described $[18,19]$. Plasmids were quantified by Nanodrop 2000c Spectrophotometer (Thermo Fisher Scientific, USA), and then transfected into 293T cells using Lipofectamine 3000 (Invitrogen, USA) according to the manufacturer's protocol. Cells were harvested $48 \mathrm{~h}$ after transfection and detected for firefly and Renilla luciferase activities using the Dual-Luciferase Reporter Assay Kit (Promega, USA) according to the manufacturer's instructions.

\section{Western Blotting Analysis}

Protein extracts were harvested by lysing the ovaries isolated from miR-17-92 cKO and control mice with RIPA lysis buffer (Beyotime, China) containing an EDTA-free protease inhibitor cocktail (BioTools, USA). The protein concentration was determined by using Bioepitope Bicinchoninic Acid protein assay kits (Bioworld, China). Equal amounts of protein (50 $\mu \mathrm{g}$ / lane) were loaded onto 12\% SDS-PAGE gels. Western blotting was performed as described [33].

The following primary antibodies were used: anti-Bmf (rabbit, 1:1, 000; Ab181148, Abcam, USA), and anti- $\beta$-tubulin (mouse, 1:100; sc-55529, Santa Cruze, USA). HRP-conjugated secondary antibodies (SA-00001-1 and SA-00001-2, Proteintech Biotechnology, USA) were used at 1:2000. The intensities of the bands were quantified using Image J software.

\section{Statistics}

All experiments were repeated at least three times. The results are presented as means \pm standard error of the mean (SEM). Means were compared using two-tailed, unpaired Student's $t$ tests using IBM SPSS statistics (v. 20.0; IBM Corp., USA); $P<0.05$ was considered statistically significant. 


\section{Cellular Physiology Cell Physiol Biochem 2018;45:491-504

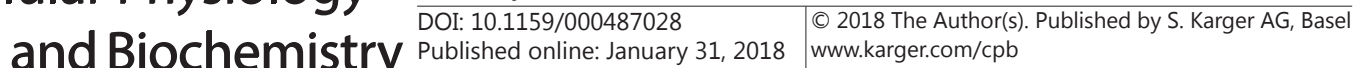 \\ Wang et al.: Ablation of the MiR-17-92 in Germ Cells Causes Subfertility}

\section{Results}

\section{Expression Levels of miR-17-92 Cluster Members in Mouse Ovaries}

The miR-17-92 cluster transcribes into a single polycistronic transcript, which yields six individual mature miRNAs, including miR-17, 18a, 19a, 20a, 19b, and 92a (Fig. 1A) [34]. According to the sequence homology and seed conservation, the six miR-17-92 components can be grouped into four distinct miRNA families, miR-17 (including miR-17 and 20a), miR18, miR-19 (including miR-19a and 19b), and miR-92 family [34] (Fig. 1A). Given the known function of the miR-17-92 cluster in regulating spermatogenesis [21-27], we predicted that it might also be essential for oogenesis. To investigate the role of miR-17-92 cluster in mouse female germ cell development, we first examined expression profiles of members of miR17-92 cluster in ovaries from 5-, 10-, 15-, 20-, 40- and 160-d-old WT mice using qRT-PCR. All members of the miR-17-92 cluster were expressed in mouse ovaries, with relatively high levels in 5- and 40-d-old mice (Fig. 1B). To further characterize the expression patterns in ovaries, we performed ISH on 8-week-old WT mouse ovaries using DIG-labeled LNA probes. The miR-17-92 cluster was expressed mainly in oocytes and ovarian stromal cells (Fig. 1C). These results suggested that the miR-17-92 cluster is involved in mouse oogenesis.

\section{The miR-17-92 cluster is Efficiently Deleted in Female Mouse Germ Cells Using Ddx4-Cre \\ Mice}

To study the functional roles of the miR-17-92 cluster in female germ cell development in vivo, we generated mouse models in which the miR-17-92 cluster was ablated genetically by applying the Cre-loxp system using $D d x 4$-Cre mice with specific Cre recombinase activity in the germ cells [28]. Ddx4-Cre mice were crossed with miR-17-92 flox/flox transgenic mice. After two rounds of crossing as shown in Fig. 2A, miR-17-92 cKO mice, with a genotype of miR-17-92 flox/4; $D d x 4$-Cre, were obtained. Because the $D d x 4$ promoter drives the expression of Cre recombinase in germ cells, the genotype of germ cells of miR-17-92 flox/+; Ddx4-Cre mice should be miR$17-92^{\Delta /+} ; D d \times 4-$

Cre, indicating deletion of miR17-92 in one allele of their germ cell genomic DNA. Consequently, the miR-17-92 cluster was deleted from the germ cells of cKO mice (miR-17-92 flox/s; Ddx4-Cre) with genomic deletion of one allele of miR-17-92 in other parts of the body. DNA from mouse tail tips was extracted to perform genotyping using appropriate primers (Fig. 2B and Table $1)$. The results of genotyping are shown in Fig. 3A.

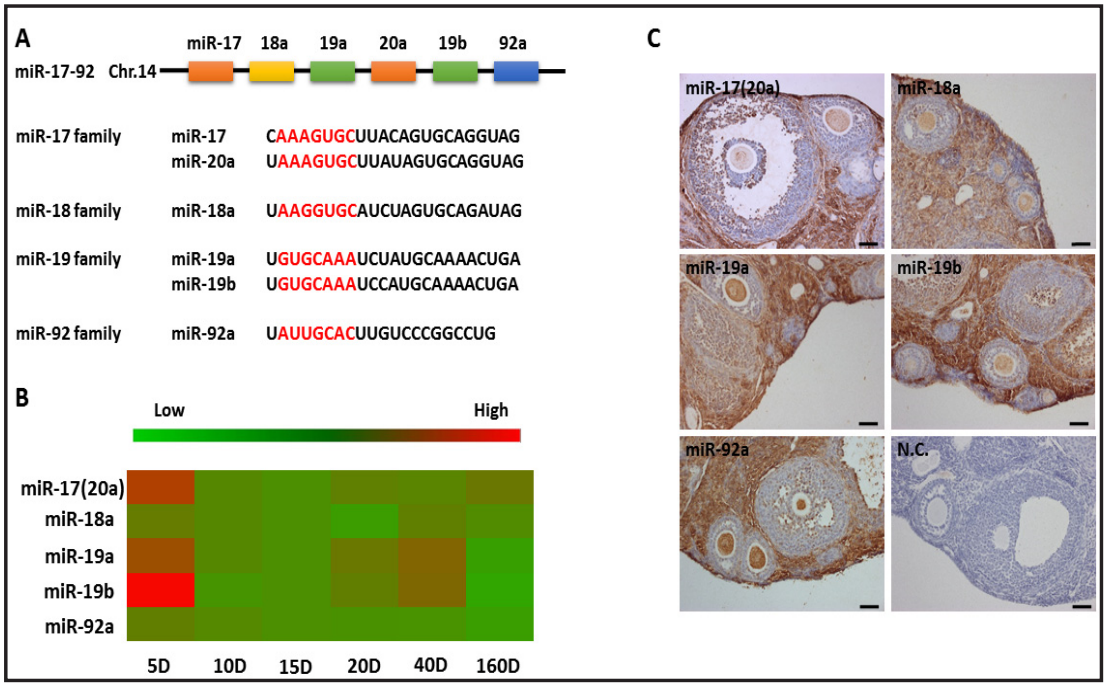

Fig. 1. The miR-17-92 cluster is expressed in the mouse ovary. (A) The genomic structure of miR-17-92 cluster on mouse chromosome 14. miRNAs with the conserved seed sequence are indicated by the same color codes. The sequence of each mature miRNA is shown, and their seed sequences are highlighted in red. (B) The relative expression levels of the miR-17-92 cluster in mouse ovaries at different postnatal stages were determined by qRT-PCR. Expression of the small RNA U6 was used as an internal standard for normalization. Primers for miR-17 also recognized miR-20a: $n=3$ per group. (C) Expression pattern of the miR-17-92 cluster was detected by ISH in 8-week-old WT mouse ovaries. The probe for miR-17 also recognized miR-20a. N.C., negative control. Scale bar, $40 \mu \mathrm{m}$. 


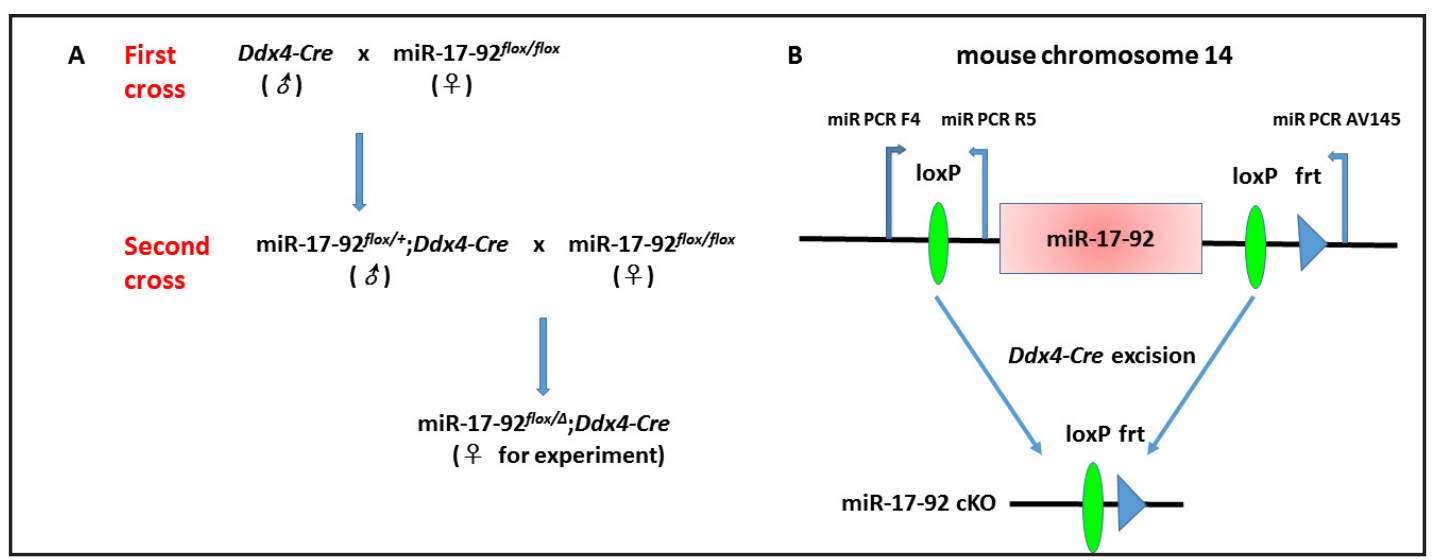

Fig. 2. The strategy of generating miR-17-92 conditional knockout mice using the Ddx4-Cre line. (A) Outline of two rounds of crossing to generate miR-17-92 conditional knockout (cKO) mice. (B) A schematic representation of deletion of miR-17-92 cluster in germ cells by using the $D d x 4$ promoter-mediated Cre transgenic mice. Primers F4, R5, and AV145 were used for genotyping.

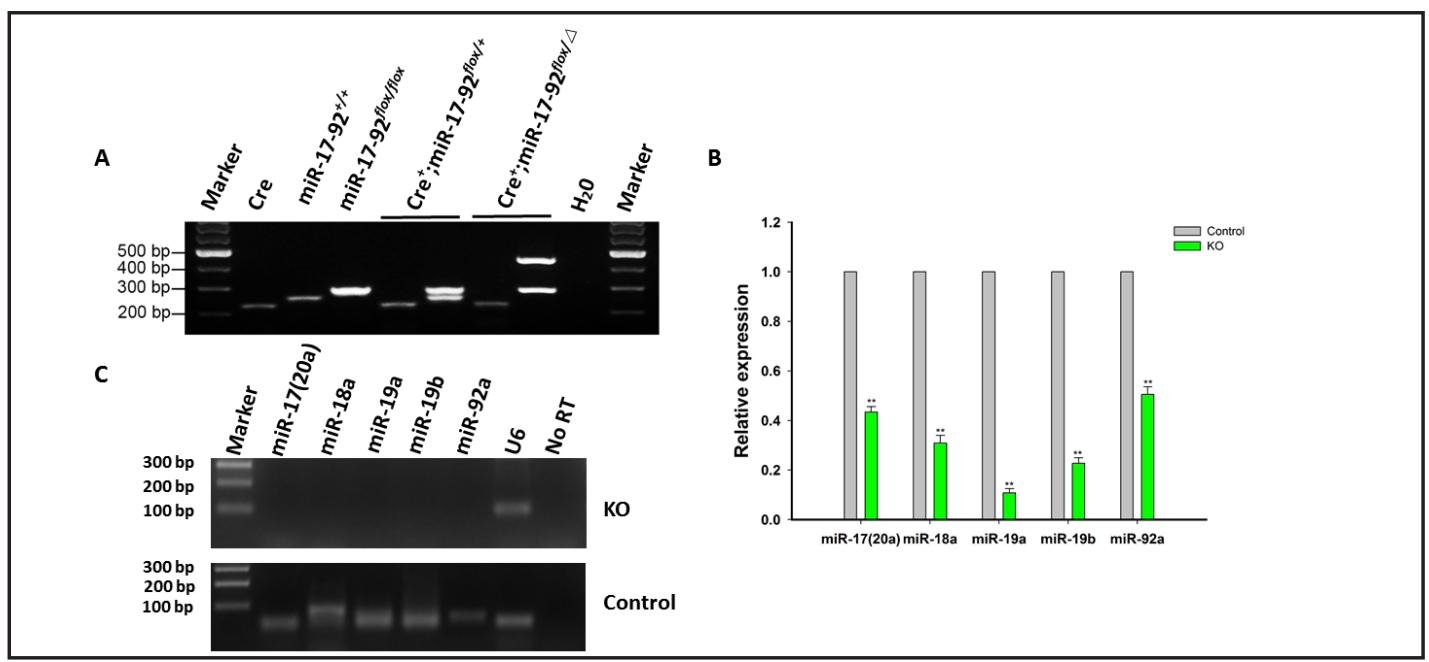

Fig. 3. Germ cell-specific deletion of miR-17-92 cluster in mice. (A) Genotyping was performed on genomic DNA extracted from tail-tip biopsies. (B) Expression levels of the individual components of miR-17-92 cluster in ovaries of miR-17-92 cKO adult mice were analyzed by qRT-PCR. $n=3$ per group. (C) Expression of individual components of the miR-17-92 cluster in oocytes of miR-17-92 cKO adult mice were analyzed by RT-PCR. KO: miR-17-92 cKO adult mice. Control: miR-17-92 flox/flox female mice with the same age of miR17-92 cKO females. ${ }^{*} \mathrm{P}<0.05,{ }^{* *} \mathrm{P}<0.01$, compared with those of controls, using two-tailed unpaired Student's t tests.

To determine the efficiency of deletion of the miR-17-92 cluster in female germ cells, ovarian tissues collected from adult cKO and control miR-17-92 flox/flox mice were subjected to qRT-PCR. As shown in Fig. 3B, the expression levels of the individual components of miR17-92 cluster were significantly downregulated in adult ovaries of cKO mice, compared with controls. Single oocytes were collected by puncturing the ovaries of cKO and control mice with a sharp needle in M2 medium (Merck, USA), selected using a mouth pipette, and then lysed further to perform RT-PCR to detect the expression of the miR-17-92 cluster as described [35]. However, no amplification band could be obtained, indicating no expression of individual components of the miR-17-92 cluster in the oocytes of cKO mice. For the oocytes of control miR-17-92 flox/flox mice, RT-PCR revealed that the miR-17-92 cluster was expressed (Fig. 3C). Our results confirmed successful germ cell depletion of the miR-17-92 cluster from miR-17-92 flox/s; Ddx4-Cre female mice. 


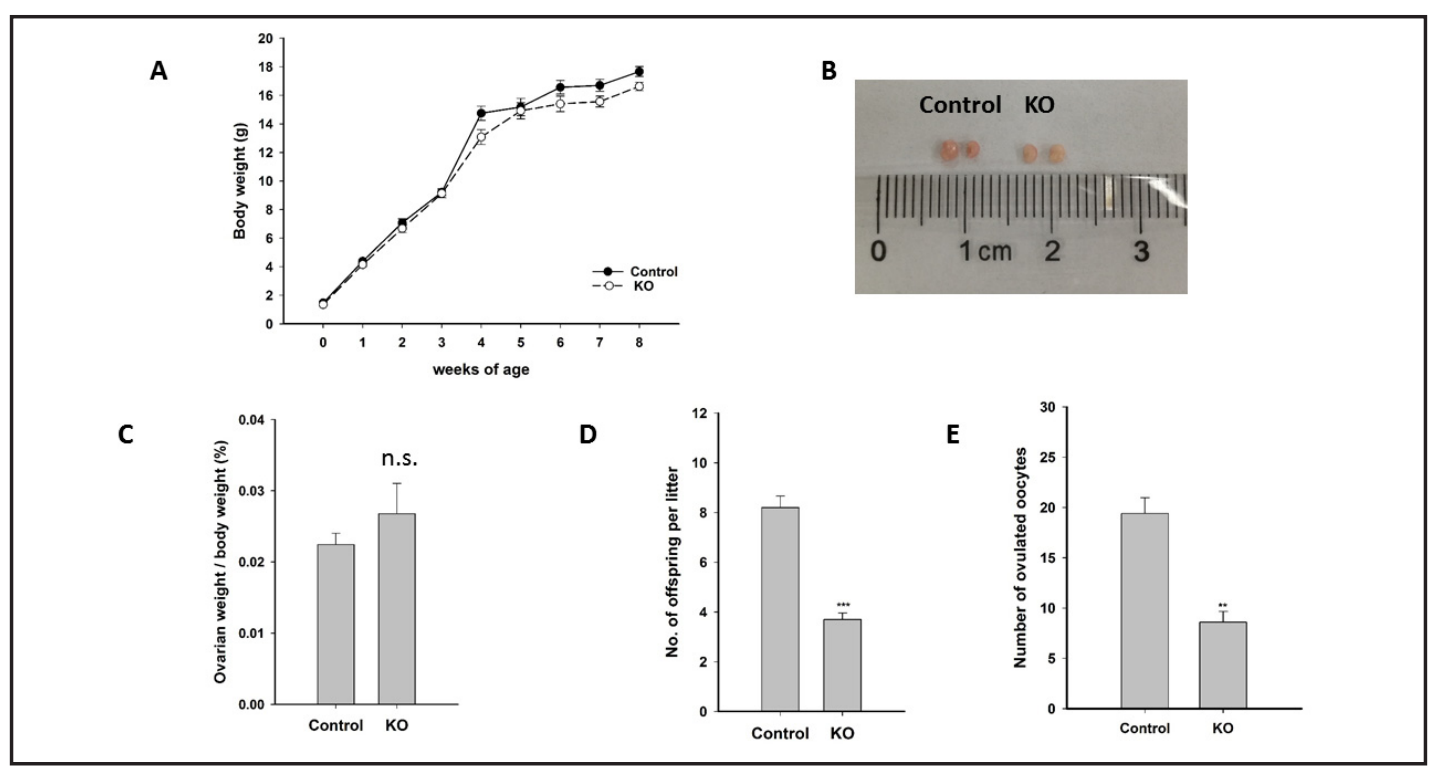

Fig. 4. Subfertility of miR-17-92 cKO adult female mice. (A) The mean body weight of miR-17-92 cKO female mice was indistinguishable from that of controls (female littermates of miR-17-92 cKO female mice). (B) Whole ovarian images of 8-week-old miR-17-92 cKO mice and control. Both left and right ovaries from one representative female mouse were shown. (C)The relative ovarian weight of miR-17-92 cKO mice was indistinguishable from controls. (D)Litter sizes from miR-17-92 cKO female mice mated with WT male mice were smaller than those of control female mice mated with WT male mice. (E)The mean number of ovulated oocytes per female mouse after hormonal stimulation. KO: miR-17-92 cKO adult mice. Control: miR-17$92^{\text {floxfflox }}$ female mice at the same age as miR-17-92 cKO females. Data are means \pm SEM, $n=3-5$ mice per group. ${ }^{*} \mathrm{P}<0.05,{ }^{* *} \mathrm{P}<0.01,{ }^{* * *} \mathrm{P}<0.001$, and n.s.: not significant.

Germ Cell Ablation of the miR-17-92 Cluster Causes Subfertility in Female Mice

The miR-17-92 $2^{\Delta / \Delta}$ newborn mice invariably died within minutes after birth [16]. The miR-17-92 null fetuses were small with severely hypoplastic lungs and clear ventricular septal defects [16]. The miR-17-92 cKO mice we generated had lost the miR-17-92 cluster from their germ cells with genomic deletion of one allele of miR-17-92 in other parts of the body. These miR-17-92 cKO female mice displayed no significant differences in body weight during the 0-8 week's postnatal development compared with control littermates (Fig. 4A). The miR-17-92 cKO mice showed normal ovarian morphology (Fig. 4B). The size of their ovaries was indistinguishable from that of controls (Fig. 4B). In addition, there was no significant difference in the ovary-to-body weight ratio between miR-17-92 cKO adult mice and controls (Fig. 4C). The fertility of 8- to 12-week-old miR-17-92 cKO female mice and controls was tested by mating experiments and the numbers of offspring per litter were recorded. Although they gave birth to heterozygous offspring when crossed with WT male mice, they displayed much reduced fecundity (Fig. 4D). Moreover, ovulated oocytes after hormonal stimulation were significantly reduced in the miR-17-92 cKO female mice compared with controls (Fig. 4E).

Because the miR-17-92 cKO female mice were subfertile, histological sections of their ovaries were examined. Many follicles contained degraded oocytes and more empty cavities were observed (Fig. 5A), suggesting that more follicles underwent atresia in the ovaries of cKO mice compared with the controls (Fig. 5B). The TUNEL assays revealed severe apoptosis in the ovaries of miR-17-92 cKO female mice compared with controls (Fig. 6A).

Follicular atresia is an apoptotic process [36, 37]. It is initiated by oocyte or granulosa cell apoptosis [38]. Thus, the expression level of apoptosis- and follicular atresia- related genes in the ovaries was evaluated. The qRT-PCR analysis revealed the expression of most 


\section{Cellular Physiology

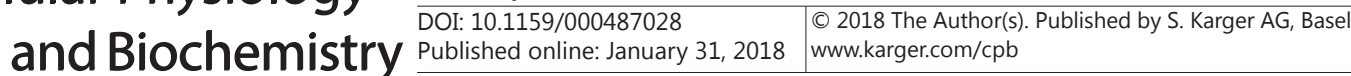

Fig. 5. Morphological comparison of ovaries from miR-17-92 cKO female mice and controls. (A) Representative images of hematoxylin staining of adult ovary sections. $\mathrm{a}$ and c: ovarian sections of control miR-17-92 flox/flox female mice; $b$ and d: ovarian sections of miR-17-92

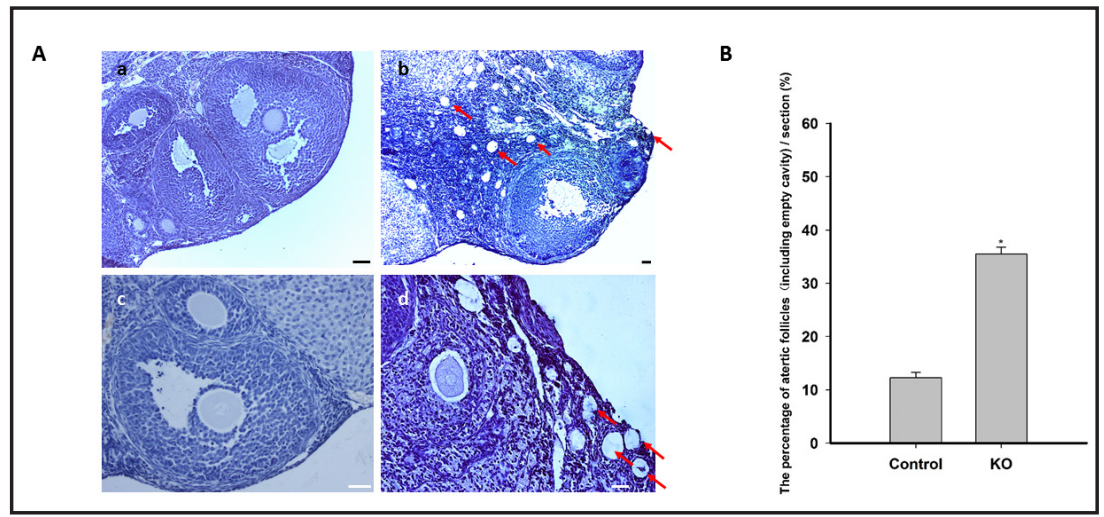
cKO mice. Scale bar, 40 $\mu \mathrm{m}$. Representative images of atretic follicles and empty cavity-like structures are indicated by arrows. (B) Statistical analysis of the percentages of atretic follicles including empty cavities in total follicles per ovarian section of miR-17-92 cKO female mice. KO: miR-17-92 cKO adult mice. Control: miR-17-92 floxfflox female mice at the same age of miR-17-92 cKO females. Data are means \pm SEM, $n=5$ mice per genotype. ${ }^{*} \mathrm{P}<0.05$.

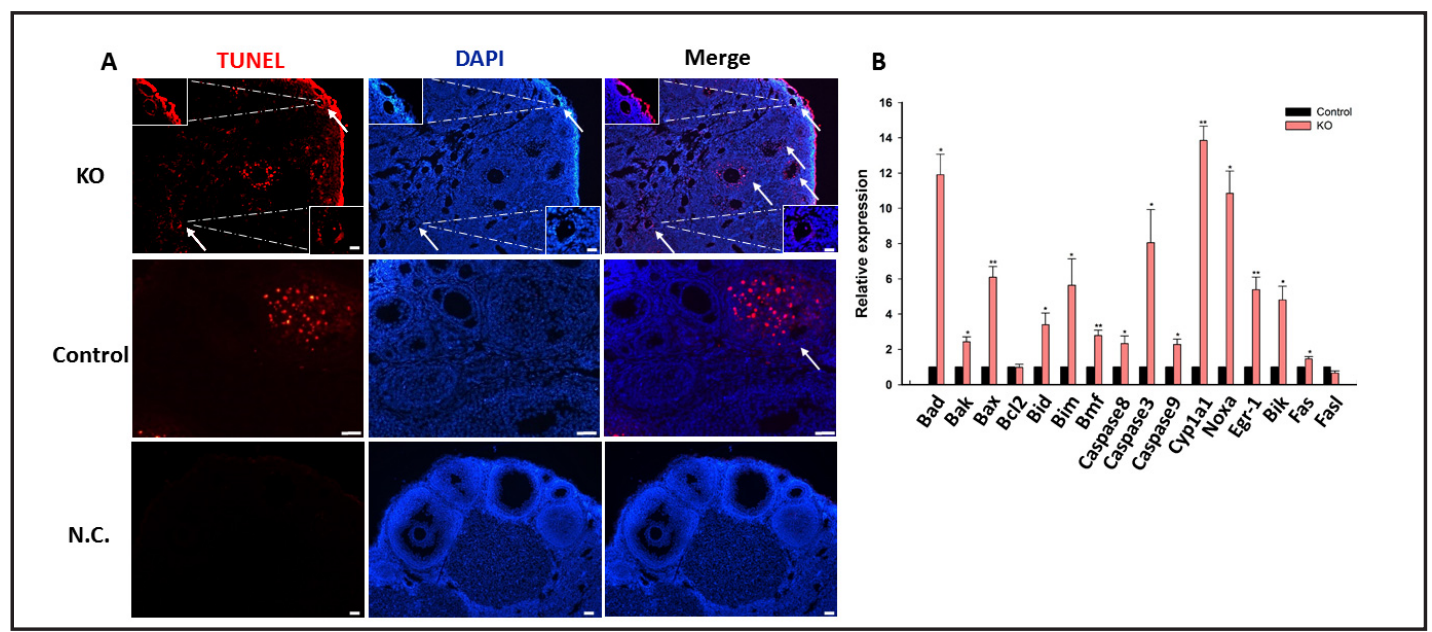

Fig. 6. Germ cell-specific deletion of the miR-17-92 cluster in mice resulted in severe cell apoptosis. (A) Detection of apoptotic cells in the ovaries of miR-17-92 cKO and control (miR-17-92 floxflox) females using TUNEL assays. N.C. negative control. Scale bars, $40 \mu \mathrm{m}$. Representative images of TUNEL-positive follicular cells are indicated by arrows and insets. (B) The expression levels of apoptosis- and follicle atresia- related genes in the ovaries of miR-17-92 cKO and control (miR-17-92 flox/flox) female mice were detected by using qRT-PCR. $\mathrm{n}=3 .{ }^{*} \mathrm{P}<0.05,{ }^{* *} \mathrm{P}<0.01$.

of the proapoptotic and follicular atresia-related genes were upregulated (Fold change $>2 ; P$ $<0.05$ ) in the ovaries of miR-17-92 cKO female mice, including Bax, Bak, Bik, Bmf, Bim, Bid, Bad, Caspase 3, Caspase 8, Caspase 9, Noxa, Cyp1a1, Egr-1. However, Fas, FasL, Bcl-2 displayed no differential expression (Fig. 6B). Previous studies have demonstrated that miR-17-92 plays an anti-apoptotic role in many physiological processes [39-41]. These results suggest that increased apoptosis induced by miR-17-92 deletion is likely one contributing factor for the more severe follicular atresia in miR-17-92 cKO female mice.

\section{Identification of miR-17-92 Targets Involved in the Aberrant Ovarian Phenotypes Observed} in miR-17-92 cKO Mice

miRNAs negatively regulate gene expression at the post-transcriptional level either by promoting degradation of the target mRNAs or by suppression of translation [2]. To further explore the direct molecular mechanisms underlying such reduced fecundity of miR-17-92 


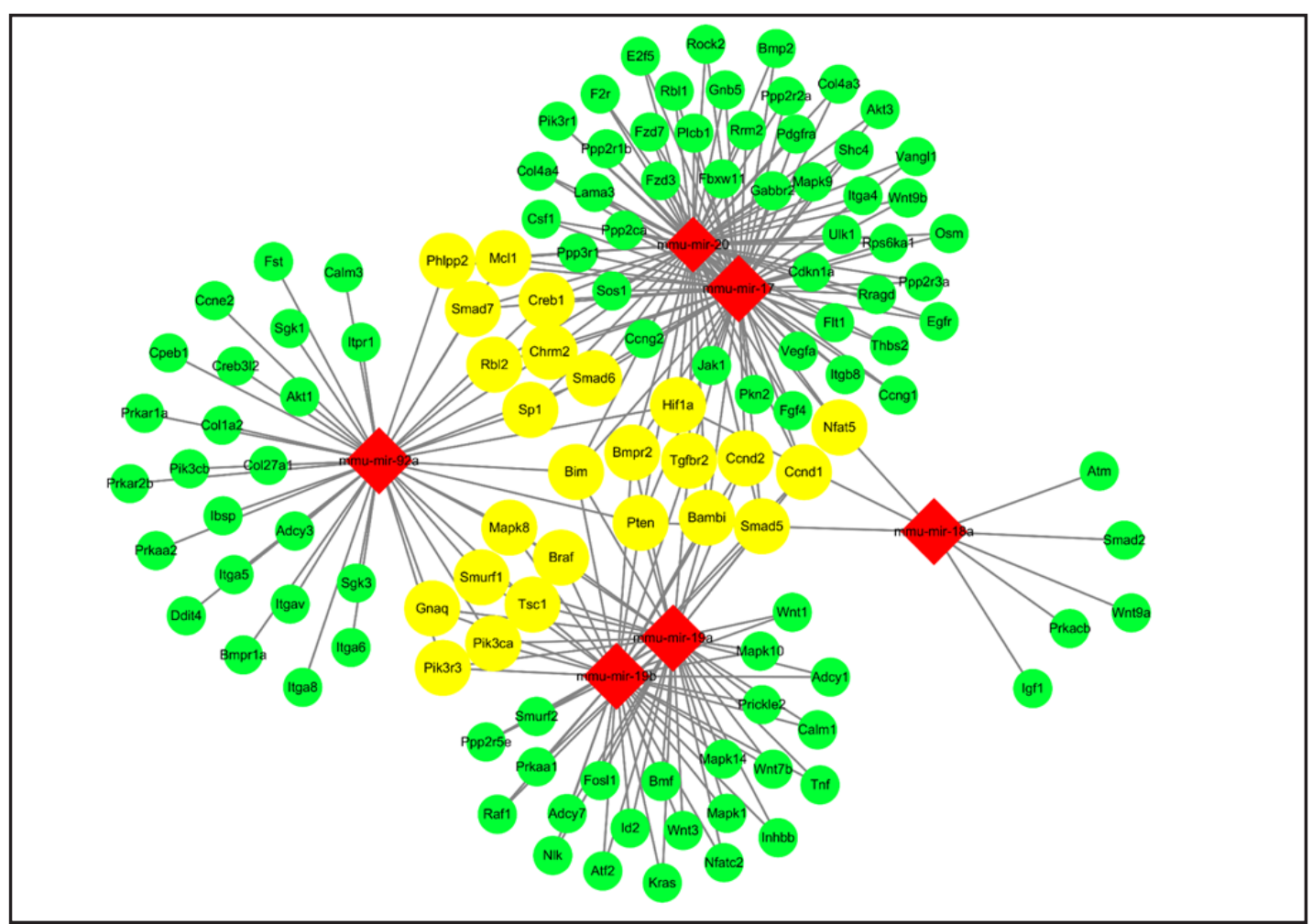

Fig. 7. The miRNA-mRNA network focused on oogenesis and cell apoptosis-related signaling pathway. Red diamond nodes represent miRNAs; green cycle nodes represent mRNAs, and yellow cycle nodes represent mRNAs which are connected by at least three edges.

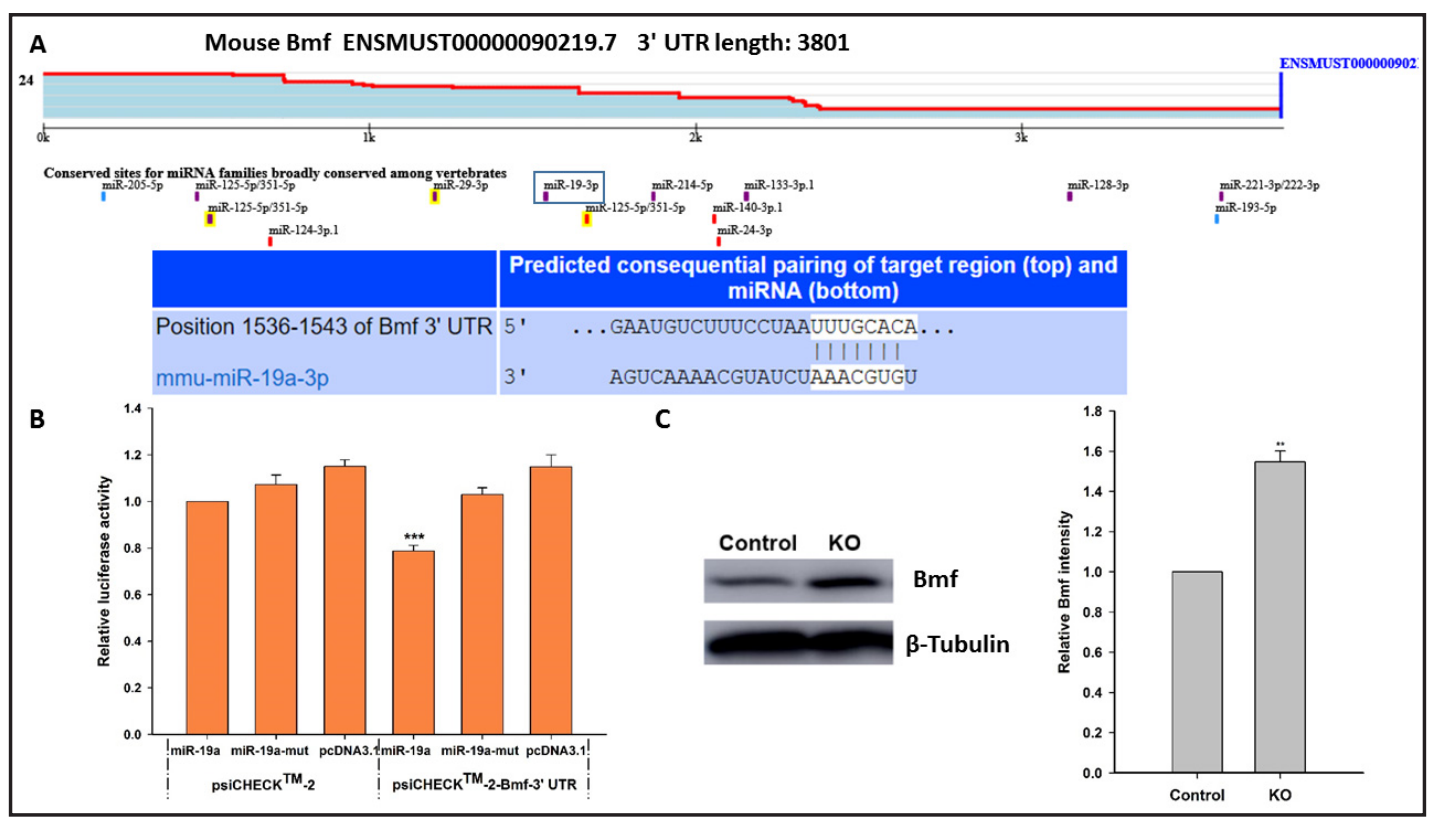

Fig. 8. Identification of $B m f$ as target gene of miR-19a. (A) A TargetScan snapshot showing the predicted targeting site of miR-19a on the 3'-UTR of Bmf. (B) Luciferase assays of miR-19a targeting effects on the Bmf 3'-UTR. miR-19a but not its mutant form (miR-19a-mut) recognized the 3'-UTR of Bmf and reduced luciferase activity. $\mathrm{n}=3,{ }^{*} \mathrm{P}<0.05,{ }^{* *} \mathrm{P}<0.01,{ }^{* *} \mathrm{P}<0.001$. (C)The protein level of BMF was increased in the ovaries of miR-17-92 cKO adult mice, detected by western blotting assays. miR-17-92 flox/flox females of the same age were used as controls. Data are presented as the mean $\pm \mathrm{SEM} ; \mathrm{n} \geq 3$ in all genotypes; ${ }^{*} \mathrm{P}<0.05$, $* * \mathrm{P}<0.01$ relative to controls.

\section{KARGER}


cKO female mice, putative target genes of the members of the miR-17-92 cluster were predicted. Three web-accessible databases, TargetScan (http://www.targetscan. org/), miRDB (http://www.mirdb.org) and miRanda (http://www.microrna.org) were searched. These predicted target genes were submitted to the KEGG pathway database (http://www.genome.jp/kegg/) for pathway enrichment analysis. Genes enriched in the canonical pathways related to follicular development and cell apoptosis were selected to construct an mRNA-miRNA interaction network (Fig. 7).

Among the target genes, $B m f$ awakened our interest. BMF is a proapoptotic protein belonging to the BH3-only subgroup of the BCL-2 family [31]. Liew et al. demonstrated that $\mathrm{Bmf}^{- \text {- }}$ mice contained more primordial and growing follicles than those from WT female mice [31]. Loss of $B m f$ also prolonged the fertile life span in female mice [31]. Vaithiyanathan et al. reported that Bmf promoted germ cell loss during murine oogenesis [30]. Therefore, the detailed

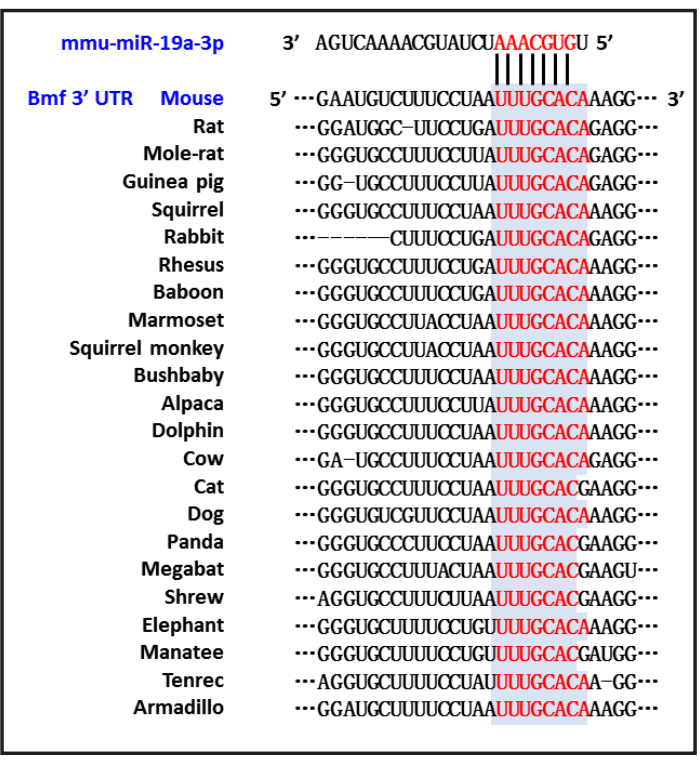

Fig. 9. Sequence alignment of the miR-19a baseparing site on the 3'-UTR of Bmf. The region complementary to the miR-19a is highly conserved among 23 species. The predicted binding sites of miR-19a with $B m f$ target sequences are highlighted in red. interaction between miR-19a and Bmf were analyzed. We found that the 3'-UTR of Bmf contains a targeting site for miR-19a (Fig. 8A). The sequence alignment between miR-19a and the $B m f 3^{\prime}$-UTR is well conserved among different species (Fig. 9). To validate the results of the algorithm prediction, a dual luciferase reporter assay was performed. The Bmf 3'-UTR was inserted into the psiCHECK ${ }^{\mathrm{TM}}$-2 dual-luciferase reporter vector and co-transfected with the miR-19a overexpression vector into $293 \mathrm{~T}$ cells. Luciferase activities were detected. While luciferase activities in constructs containing the 3'-UTR of Bmf were not affected by the mutated miR-19a or control empty vectors, they were significantly reduced by the expression of miR-19a. The dual luciferase experiments confirmed that miR-19a could bind to the 3'-UTR sequence of Bmf (Fig. 8B). Furthermore, qRT-PCR and western blot analysis revealed increased expression of the $B m f$ in the ovaries of miR-17-92 cKO female mice, indicating that this gene is a miR-19a target and is negatively regulated by miR-19a (Fig. 6B and $8 \mathrm{C}$ ). Our results suggest a specific targeting regulation by miR-19a on Bmf activity in the ovaries of miR-17-92 cKO female mice, contributing to the phenotype of follicular atresia.

\section{Discussion}

Oogenesis is a precise biological process and any disorder in it will result in female infertility. Identification of specific molecules that regulate oogenesis would contribute to our better understanding of the pathophysiology of infertility caused by oogenesis disorders. Emerging studies have shown that, like coding genes, miRNAs play critical roles in germ cell development $[42,14]$. Gene KO mouse models are powerful tools to gain insight into the involvement of miRNAs in the function of germ cells and their contribution to gametogenesis. We generated mouse models in which the miR-17-92 cluster was genetically ablated in germ cells by applying the Cre-loxp system. Even though the miR-17-92 cKO female mice gave birth to heterozygous offspring when crossed with WT male mice, they displayed much reduced 


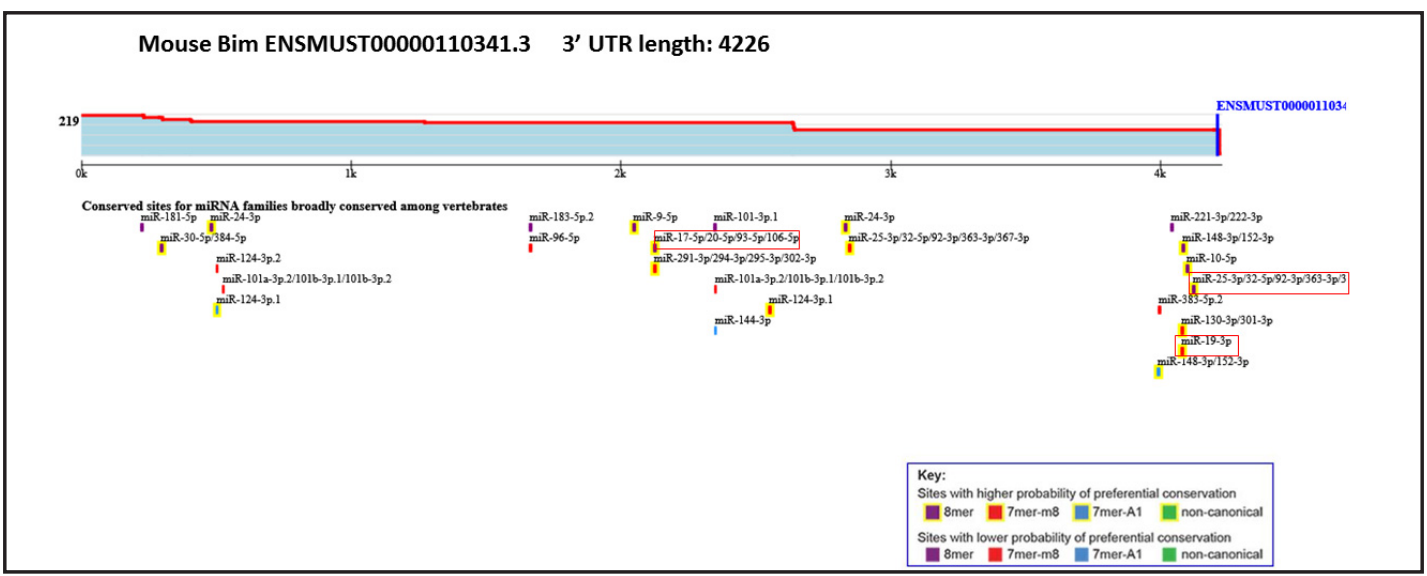

Fig. 10. A TargetScan snapshot showing the predicted targeting sites of miR-17, miR-19a, miR-19b, miR-20a and miR-92a on the 3'-UTR of Bim.

fecundity. These mice show subfertility rather than infertility, which is likely because miRNAs negatively regulate target gene expressions post-transcriptionally by fine tuning [14].

Lower ovulation numbers and increased follicular atresia were observed in the cKO female mice than in controls. It is believed that apoptosis is the major mechanism involved in follicular atresia. There are two apoptotic pathways in mammalian cells: the intrinsic pathway (also known as the mitochondrial control of apoptosis signaling pathway) and the extrinsic apoptotic pathway [43]. Several studies have suggested that germ cell apoptosis is mediated by the intrinsic apoptosis pathway [44], which is regulated by the relative levels and activities of pro- and anti-apoptotic members of the BCL2 protein family [43]. The expression of pro-apoptotic BH3-only genes (Noxa, Bmf, Bid, Bik, Bad and Bim), the proapoptotic effector protein gene (Bax and Bak), initiator caspases (Caspase 8 and Caspase 9) and executioner caspase (Caspase 3) were upregulated in the ovaries of miR-17-92 cKO female mice. Moreover, some follicular atresia-related genes (Cyp1a1 and Egr-1) were also upregulated. However, Fas, Fasl and Bcl-2 displayed no differential expression. These data support the hypothesis that deletion of miR-17-92 cluster in the germ cells results in severe follicle atresia via the intrinsic apoptotic pathway.

When considering the physiology of follicular atresia, one might expect genes for apoptosis to be inversely regulated by miRNAs. Here we identified $B m f$ as a target gene of the miR-17-92 cluster in the cKO mouse ovaries. Likewise, Bim (also known as Bcl2l11) also belongs to the pro-apoptotic BH3-only BCL-2 family member, and it acts as an apoptotic activator [40]. The 3'-UTR of Bim contains targeting sites for miR-17, miR-19a, miR-19b, miR-20a and miR-92a (Fig. 10). Previous studies identified Bim as direct targets of miR-17 $[40,45]$, miR-20a [27, 40], and miR-92a [40]. Xie et al. identified Bim as the direct target of miR-20a in the testes of miR-17-92 cKO male mice, and demonstrated that it is implicated in the process of spermatogenesis [27]. Moreover, Wang et al. reported Bim induces porcine follicular atresia [46]. qRT-PCR analysis revealed the increased expression of Bim in the ovaries of miR-17-92 cKO female mice, suggesting that Bim acts as the target gene of miR17-92 in cKO mice ovaries, contributing to follicular atresia. The elevated oocyte degradation and follicular atresia in the ovaries of miR-17-92 cKO female mice appears to be at least in part due to upregulated expression of $B m f$ (a putative target of miR-19a) and Bim (a putative target of miR-17, miR-19a, miR-19b, miR-20a and miR-92a). Thus, the normal regulatory function of the miR-17-92 cluster is to promote oocyte survival. These results are consistent with previous reports that miR-17-92 plays an anti-apoptotic role in many physiological processes [39-41].

miRNAs are frequently transcribed together as a polycistron unit that is processed into multiple individual mature miRNAs [34]. The miR-17-92 cluster is a typical example yielding 


\section{Cellular Physiology Cell Physiol Biochem 2018:45:491-504 \begin{tabular}{l|l} 
and Biochemistry Published onlIne: January 31, 2018 & $\begin{array}{l}\text { (c) 2018 The Author(s). Published by S. Karger AG, Basel } \\
\text { www.karger.com/cpb }\end{array}$
\end{tabular}}

Wang et al.: Ablation of the MiR-17-92 in Germ Cells Causes Subfertility

six individual mature miRNAs belonging to four distinct miRNA families [34]. Moreover, a single miRNA can simultaneously target multiple genes. Therefore, it is possible that there might be more genes directly affected by the miR-17-92 cluster involved in oogenesis other than Bim and Bmf. We intend to identify more target genes and construct an interaction network for miR-17-92 and its targets, which collaborate to regulate the process of oogenesis.

In this study we have demonstrated an essential role of the miR-17-92 cluster in regulating oogenesis. Deletion of this cluster in germ cells of female mice caused increased oocyte degradation and follicular atresia, perturbed proper oogenesis, and ultimately resulted in subfertility. Our results reveal an important molecular mechanism of miR-17-92 in modulating murine oogenesis by targeting $B m f$, which promotes germ cell loss during murine oogenesis [30]. These findings will assist in better understanding the etiology of disorders in oogenesis and in developing new therapeutic targets for female infertility.

\section{Acknowledgements}

This work was supported by National Basic Research Program of China (2017YFA0504201) and the National Nature Science Foundation of China (81720108017).

\section{Disclosure Statement}

The authors declare that they have no conflict of interest.

\section{References}

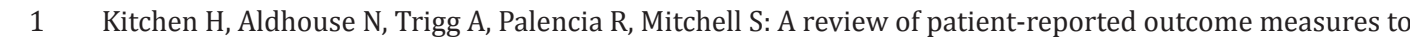
assess female infertility-related quality of life. Health Qual Life Outcomes 2017;15:86.

2 Ambros V: The functions of animal microRNAs. Nature 2004;431:350-355.

-3 Bartel DP: MicroRNAs: genomics, biogenesis, mechanism, and function. Cell 2004;116:281-297.

4 Plasterk RH: Micro RNAs in animal development. Cell 2006;124:877-881.

5 Ro S, Song R, Park C, Zheng H, Sanders KM, Yan W: Cloning and expression profiling of small RNAs expressed in the mouse ovary. RNA 2007;13:2366-2380.

6 Ahn HW, Morin RD, Zhao H, Harris RA, Coarfa C, Chen ZJ, Milosavljevic A, Marra MA, Rajkovic A: MicroRNA transcriptome in the newborn mouse ovaries determined by massive parallel sequencing. Mol Hum Reprod 2010;16:463-471.

7 Liang Y, Ridzon D, Wong L, Chen C: Characterization of microRNA expression profiles in normal human tissues. BMC Genomics 2007;8:166.

-8 Miles JR, McDaneld TG, Wiedmann RT, Cushman RA, Echternkamp SE, Vallet JL, Smith TP: MicroRNA expression profile in bovine cumulus-oocyte complexes: possible role of let-7 and miR-106a in the development of bovine oocytes. Anim Reprod Sci 2012;130:16-26.

-9 Ling YH, Ren CH, Guo XF, Xu LN, Huang YF, Luo JC, Zhang YH, Zhang XR, Zhang ZJ: Identification and characterization of microRNAs in the ovaries of multiple and uniparous goats (Capra hircus) during follicular phase. BMC Genomics 2014;15:339.

10 Di R, He J, Song S, Tian D, Liu Q, Liang X, Ma Q Sun M, Wang J, Zhao W, Cao G, Wang J, Yang Z, Ge Y, Chu M: Characterization and comparative profiling of ovarian microRNAs during ovine anestrus and the breeding season. BMC Genomics 2014;15:899.

11 Li M, Liu Y, Wang T, Guan J, Luo Z, Chen H, Wang X, Chen L, Ma J, Mu Z, Jiang AA, Zhu L, Lang Q Zhou X, Wang J, Zeng W, Li N, Li K, Gao X, Li X: Repertoire of porcine microRNAs in adult ovary and testis by deep sequencing. Int J Biol Sci 2011;7:1045-1055.

12 Hong X, Luense LJ, McGinnis LK, Nothnick WB, Christenson LK: Dicer1 is essential for female fertility and normal development of the female reproductive system. Endocrinology 2008;149:6207-6212. 


\section{Cellular Physiology Cell Physiol Biochem 2018;45:491-504

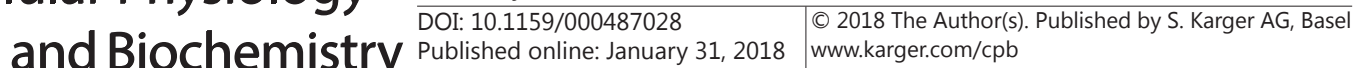 \\ Wang et al.: Ablation of the MiR-17-92 in Germ Cells Causes Subfertility}

13 Lei L, Jin S, Gonzalez G, Behringer RR, Woodruff TK: The regulatory role of Dicer in folliculogenesis in mice. Mol Cell Endocrinol 2010;315:63-73.

14 Maalouf SW, Liu WS, Pate JL: MicroRNA in ovarian function. Cell Tissue Res 2016;363:7-18.

15 Mendell JT: miRiad roles for the miR-17-92 cluster in development and disease. Cell 2008;133:217-222.

16 Ventura A, Young AG, Winslow MM, Lintault L, Meissner A, Erkeland SJ, Newman J, Bronson RT, Crowley D, Stone JR, Jaenisch R, Sharp PA, Jacks T: Targeted deletion reveals essential and overlapping functions of the miR-17 through 92 family of miRNA clusters. Cell 2008;132:875-886.

17 Chen J, Huang ZP, Seok HY, Ding J, Kataoka M, Zhang Z, Hu X, Wang G, Lin Z, Wang S, Pu WT, Liao R, Wang DZ: mir-17-92 cluster is required for and sufficient to induce cardiomyocyte proliferation in postnatal and adult hearts. Circ Res 2013;112:1557-1566.

18 Bian S, Hong J, Li Q Schebelle L, Pollock A, Knauss JL, Garg V, Sun T: MicroRNA cluster miR-17-92 regulates neural stem cell expansion and transition to intermediate progenitors in the developing mouse neocortex. Cell Rep 2013;3:1398-1406.

19 Jin J, Kim SN, Liu X, Zhang H, Zhang C, Seo JS, Kim Y, Sun T: miR-17-92 Cluster Regulates Adult Hippocampal Neurogenesis, Anxiety, and Depression. Cell Rep 2016;16:1653-1663.

20 Xiang J, Wu J: Feud or Friend? The Role of the miR-17-92 Cluster in Tumorigenesis. Curr Genomics 2010;11:129-135.

21 Novotny GW, Sonne SB, Nielsen JE, Jonstrup SP, Hansen MA, Skakkebaek NE, Rajpert-De Meyts E, Kjems J, Leffers H: Translational repression of E2F1 mRNA in carcinoma in situ and normal testis correlates with expression of the miR-17-92 cluster. Cell Death Differ 2007;14:879-882.

22 Hayashi K, Chuva de Sousa Lopes SM, Kaneda M, Tang F, Hajkova P, Lao K, O’Carroll D, Das PP, Tarakhovsky A, Miska EA, Surani MA: MicroRNA biogenesis is required for mouse primordial germ cell development and spermatogenesis. PLoS One 2008;3:e1738.

-23 Lian J, Zhang X, Tian H, Liang N, Wang Y, Liang C, Li X, Sun F: Altered microRNA expression in patients with non-obstructive azoospermia. Reprod Biol Endocrinol 2009;7:13.

-24 Bjork JK, Sandqvist A, Elsing AN, Kotaja N, Sistonen L: miR-18, a member of Oncomir-1, targets heat shock transcription factor 2 in spermatogenesis. Development 2010;137:3177-3184.

25 Tong MH, Mitchell DA, McGowan SD, Evanoff R, Griswold MD: Two miRNA clusters, Mir-17-92 (Mirc1) and Mir-106b-25 (Mirc3), are involved in the regulation of spermatogonial differentiation in mice. Biol Reprod 2012;86:72.

26 He Z, Jiang J, Kokkinaki M, Tang L, Zeng W, Gallicano I, Dobrinski I, Dym M: MiRNA-20 and mirna-106a regulate spermatogonial stem cell renewal at the post-transcriptional level via targeting STAT3 and Ccnd1 Stem Cells 2013;31:2205-2217.

-27 Xie R, Lin X, Du T, Xu K, Shen H, Wei F, Hao W, Lin T, Lin X, Qin Y, Wang H, Chen L, Yang S, Yang J, Rong X, Yao K, Xiao D, Jia J, Sun Y: Targeted Disruption of miR-17-92 Impairs Mouse Spermatogenesis by Activating mTOR Signaling Pathway. Medicine (Baltimore) 2016;95:e2713.

28 Gallardo T, Shirley L, John GB, Castrillon DH: Generation of a germ cell-specific mouse transgenic Cre line, Vasa-Cre. Genesis 2007;45:413-417.

-29 Obernosterer G, Martinez J, Alenius M: Locked nucleic acid-based in situ detection of microRNAs in mouse tissue sections. Nat Protoc 2007;2:1508-1514.

30 Vaithiyanathan K, Liew SH, Zerafa N, Gamage T, Cook M, O’Reilly LA, Bouillet P, Scott CL, Strasser A, Findlay JK, Hutt KJ: BCL2-modifying factor promotes germ cell loss during murine oogenesis. Reproduction 2016;151:553-562.

31 Liew SH, Vaithiyanathan K, Cook M, Bouillet P, Scott CL, Kerr JB, Strasser A, Findlay JK, Hutt KJ: Loss of the proapoptotic BH3-only protein BCL-2 modifying factor prolongs the fertile life span in female mice. Biol Reprod 2014;90:77.

-32 Sun J, Lin Y, Wu J: Long non-coding RNA expression profiling of mouse testis during postnatal development. PLoS One 2013;8:e75750.

33 Sun J, Wang J, He L, Lin Y, Wu J: Knockdown of polycomb-group RING finger 6 modulates mouse male germ cell differentiation in vitro. Cell Physiol Biochem 2015;35:339-352.

34 Olive V, Li Q, He L: mir-17-92: a polycistronic oncomir with pleiotropic functions. Immunol Rev 2013;253:158-166. 


\section{Cellular Physiology Cell Physiol Biochem 2018;45:491-504 and Biochemistry \begin{tabular}{l|l} 
DOI: 10.1159/000487028 \\
Published 2018 (O) 2018 The Author(s). Published by S. Karger AG, Basel \\
wwww.karger.com/cpb
\end{tabular} \\ Wang et al.: Ablation of the MiR-17-92 in Germ Cells Causes Subfertility}

-35 Ding X, Liu G, Xu B, Wu C, Hui N, Ni X, Wang J, Du M, Teng X, Wu J: Human GV oocytes generated by mitotically active germ cells obtained from follicular aspirates. Sci Rep 2016;6:28218.

-36 Hughes FM, Jr., Gorospe WC: Biochemical identification of apoptosis (programmed cell death) in granulosa cells: evidence for a potential mechanism underlying follicular atresia. Endocrinology 1991;129:24152422.

-37 De Felici M: Ovarian follicular atresia: a model for apoptosis. Cell Death Differ 1997;4:260-261.

-38 Reynaud K, Driancourt MA: Oocyte attrition. Mol Cell Endocrinol 2000;163:101-108.

-39 Liu J, Yao W, Yao Y, Du X, Zhou J, Ma B, Liu H, Li Q, Pan Z: MiR-92a inhibits porcine ovarian granulosa cell apoptosis by targeting Smad7 gene. FEBS Lett 2014;588:4497-4503.

40 Guo L, Xu J, Qi J, Zhang L, Wang J, Liang J, Qian N, Zhou H, Wei L, Deng L: MicroRNA-17-92a upregulation by estrogen leads to Bim targeting and inhibition of osteoblast apoptosis. J Cell Sci 2013;126:978-988.

41 Yan HL, Xue G, Mei Q, Wang YZ, Ding FX, Liu MF, Lu MH, Tang Y, Yu HY, Sun SH: Repression of the miR-17-92 cluster by 553 has an important function in hypoxia-induced apoptosis. EMBO J 2009;28:2719-2732.

42 Grossman H, Shalgi R: A Role of MicroRNAs in Cell Differentiation During Gonad Development. Results Probl Cell Differ 2016;58:309-336.

43 Hutt KJ: The role of BH3-only proteins in apoptosis within the ovary. Reproduction 2015;149:R81-89.

44 Myers M, Morgan FH, Liew SH, Zerafa N, Gamage TU, Sarraj M, Cook M, Kapic I, Sutherland A, Scott CL, Strasser A, Findlay JK, Kerr JB, Hutt KJ: PUMA regulates germ cell loss and primordial follicle endowment in mice. Reproduction 2014;148:211-219.

45 Yan HJ, Liu WS, Sun WH, Wu J, Ji M, Wang Q, Zheng X, Jiang JT, Wu CP: miR-17-5p inhibitor enhances chemosensitivity to gemcitabine via upregulating Bim expression in pancreatic cancer cells. Dig Dis Sci 2012;57:3160-3167.

-46 Wang XL, Wu Y, Tan LB, Tian Z, Liu JH, Zhu DS, Zeng SM: Follicle-stimulating hormone regulates proapoptotic protein Bcl-2-interacting mediator of cell death-extra long (BimEL)-induced porcine granulosa cell apoptosis. J Biol Chem 2012;287:10166-10177. 